NISSUNA UMANA INVESTIGAZIONE SI PUO DIMANDARE VERA SCIENZIA S'ESSA NON PASSA PER LE MATEMATICHE DIMOSTRAZIONI LEONARDO DA VINCI

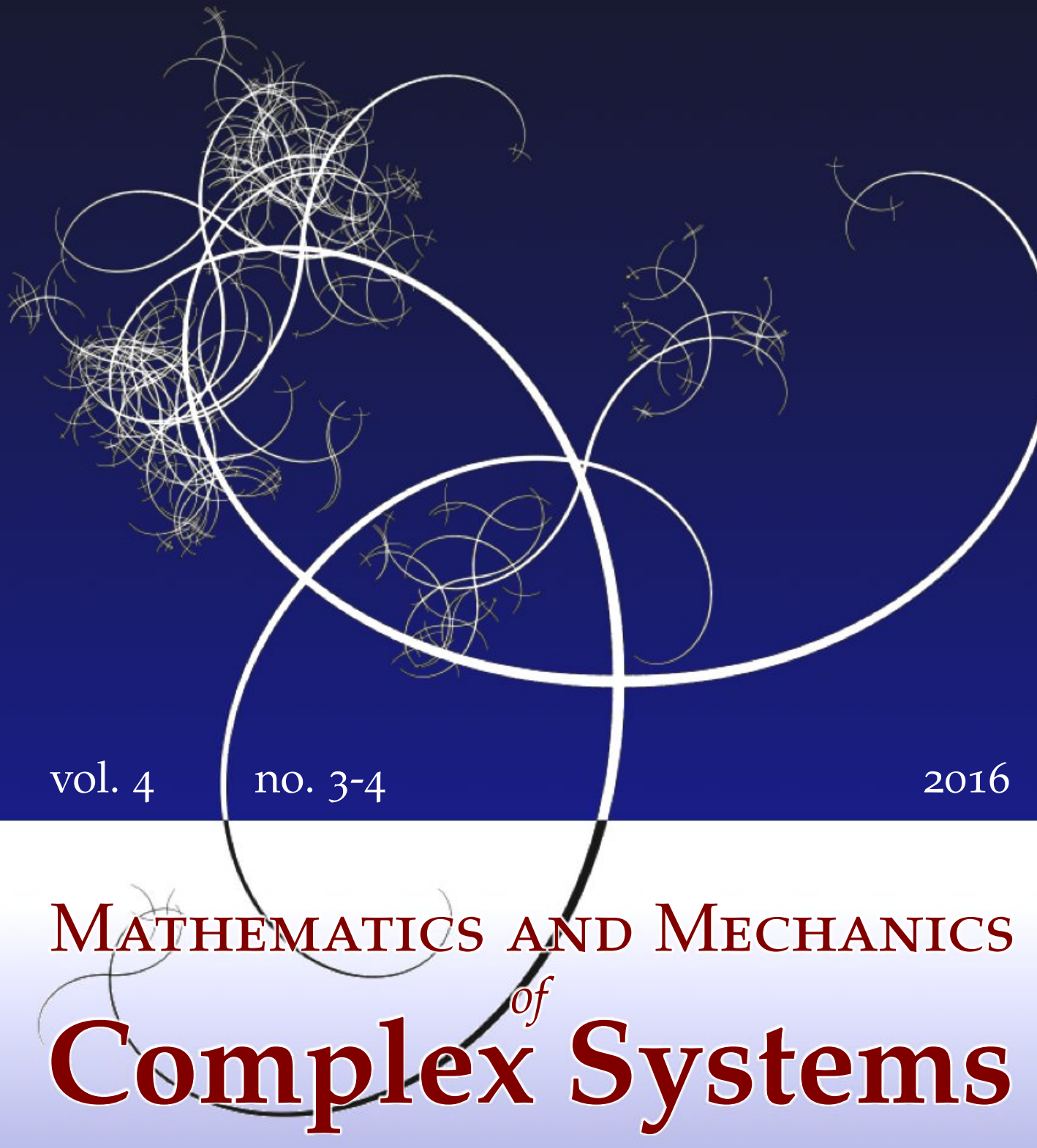

VINCENZO GRECCHI

QUANTUM MECHANICS:

SOME BASIC TECHNIQUES FOR SOME BASIC MODELS

I: THE MODELS 


\title{
QUANTUM MECHANICS: \\ SOME BASIC TECHNIQUES FOR SOME BASIC MODELS \\ I: THE MODELS
}

\author{
VINCENZO GRECCHI
}

\begin{abstract}
This is a short review of the main results on the oscillators considered basic models in quantum mechanics. Since the potential is polynomial, it is possible to extend the stationary states to entire functions on the complex plane where the semiclassical theory works better. The control on the energy levels is based on the isolation of the nodes, the zeros stable at the unperturbed limit. A new and simple model for the process of racemization of chiral molecules is added.
\end{abstract}

\section{Introduction}

For me it is a great pleasure to contribute to a deserved homage to Professor Lucio Russo. I have to offer only this flower from my garden, a review of results on the spectrum of basic operators in quantum mechanics.

I remember conversations with Lucio about the existence of gambling experts ( $\varkappa \cup \beta \varepsilon \cup \tau \varkappa \circ$ ) in Roman times, as reported by Plotinus (204-270 AD), who regarded them as professional scientists. ${ }^{1}$ Does this mean there were probabilist mathematicians in those times? In any case, it seems that only discrete probability was known, since Plotinus apparently rejected the possibility of anything like what we call statistical mechanics:

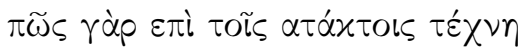

(what science can operate where there is no order?) ${ }^{2}$

This memory is appropriate as an introduction, since probability theory was Lucio's first interest and it is one of the bases of quantum mechanics.

The reason for this review is the difficulty of reading all the single papers considered here. The problems considered in such papers are not unrelated and need

\section{Communicated by Francesco dell'Isola.}

PACS2010: 03.65.-w.

Keywords: quantum oscillators, $P T$ symmetry, isospectral Hamiltonians, spectral analysis, analyticity of the eigenvalues.

${ }^{1}$ Enneads, III, 1, 6, lines 9-10.

${ }^{2}$ Enneads, III, 1, 3, line 15. 
the same techniques to be solved. In particular, I recall the methods for summing divergent series, going back to Guido Grandi [1710].

This review is almost entirely restricted to one-dimensional problems with polynomial potentials (oscillators), with a focus on the cubic case.

Among the points of interest in such research is the meaning of perturbation series in quantum field theory, where the summability test for perturbation series is relevant.

Also of interest from the viewpoint of rigorous atomic and molecular physics are the possible models for molecular structures. In some quantum models presented here there are resonances and associated metastable states. The notion of resonance in quantum mechanics is similar to the same notion in acoustics as discussed by Pietro Mengoli [1670] (see also [Martinez and Nédélec 2012]).

I recall that Lucio has studied Mengoli's contribution to the beginnings of modern mathematical analysis.

All the molecules with a structure [Woolley 1982] and the atoms in an external uniform electric field (the Stark effect) are in a metastable state [Graffi and Grecchi 1978].

In the case of an analytic potential, a resonance is a complex eigenvalue of a non-self-adjoint operator related in a definite way to the original Hamiltonian of the model [Aguilar and Combes 1971].

A particular kind of non-self-adjoint operator is known as PT or PT-symmetric. An operator $H$ is PT if $(P T) H(P T)^{-1}=H$, where $P$ and $T$, the parity and timereversal operators, are defined in (2) below. New interest in such problems started from a collaboration with the late professor Vladimir Buslaev of Saint Petersburg. In [Buslaev and Grecchi 1993] we solved a conjecture of Zinn-Justin about the coincidence of two perturbation series: one in a double-well problem and the other in an unstable single-well problem (where there are expected resonances). We proved the isospectrality of the Hamiltonian (coincidence of the eigenvalues) of the first problem with a PT extension of the formal Hamiltonian of the other one.

Actually also the operator for the resonances of the linear potential (free Stark effect), previously considered by Herbst and Simon [1978], and an analytic continuation of the operator for the resonances in the sense of Gamow [Caliceti and Maioli 1983] of the cubic potential, previously considered by Caliceti, Graffi and Maioli [Caliceti et al. 1980], are PT operators.

I have considered analytic continuations of the resonances of the cubic oscillator in collaboration with André Martinez and Marco Maioli [Grecchi and Martinez 2013; Grecchi et al. 2009; 2010] and with Riccardo Giachetti [Giachetti and Grecchi 2011]. In this last study, we proved the existence of level crossings in a doublewell problem. During the crossing process, the corresponding states pass from the bilocalization in both the wells to the localization in a single well (not the same 
for the two states). This is the proof of the existence of singularities (similar to the Bender-Wu singularities of the levels of the quartic oscillator [Bender and $\mathrm{Wu}$ 1969; Harrell and Simon 1980; Benassi and Grecchi 1980; Alvarez 1995]) of the resonances as analytic functions of the parameter.

Section 2 recaps the essentials of quantum mechanics to fix some notions. In Section 3 we discuss solvable oscillators as examples. In Section 4 is a short exposition on the quartic oscillator. Section 5 briefly cover PT oscillators. Section 6 is devoted to the cubic oscillator with a single well. In Section 7 is the cubic PT oscillator with a double well. In Section 8 a simple model for the racemization effect is discussed.

\section{Essentials of quantum mechanics}

Sir William Rowan Hamilton [1834; 1835] laid the basis for the understanding tht classical mechanics, in the Hamilton-Jacobi formalism, is a short-wavelength limit of a possible theory of matter waves. Actually, he obtained the Hamilton-Jacobi method in mechanics by analogy with the eikonal approximation of the equations of electromagnetic waves.

Schrödinger (1926) formalized this theory, quantum mechanics, by his famous equation.

In $d$-dimensional quantum mechanics, the Hamiltonian of a particle of mass $m=\frac{1}{2}$ is a linear operator in $\mathscr{H}=L^{2}\left(\mathbb{R}^{d}\right)$ of the kind

$$
H=p^{2}+V, \quad \text { where } p^{2}=-\nabla^{2}
$$

and where the Planck constant $\hbar=h / 2 \pi$ is unitary and $V$ is the formal multiplication operator by the real function $V(x)$ defined by $(V \psi)(x)=V(x) \psi(x)$ for a vector $\psi \in \mathscr{H}$ represented by a function $\psi(x)$. In the sequel the multiplication operator $V$ is identified with its corresponding function $V(x)$. We call unstable a model with a potential not bounded below at infinity. The basic operators of interest are self-adjoint (s.a.) (observables) restricted to their domains [Kato 1966]. The possible eigenvalues of a self-adjoint operator are necessarily real. We call $D(A)$ the domain of the operator $A$, and the domain of the Hamiltonian $H$, defined as a sum of two operators by (1), satisfies the condition

$$
D(V) \cap D\left(p^{2}\right) \subset D(H) \subset \mathscr{H} .
$$

The spectral theorem [Kato 1966; Reed and Simon 1975; 1978] allows one to associate the self-adjoint Hamiltonian with the real energy spectrum. A state is a class of equivalence of vectors $\psi \in \mathscr{H}$ of unitary norm, with $\psi \sim \lambda \psi,|\lambda|=1$. At any $t \in \mathbb{R}$ the vector becomes $\psi(t)=U_{t} \psi$, where $U_{t}:=\exp (-i t H)$ is a unitary operator conserving the norm. We define a (energy) level to be an eigenvalue $E$ 
and a stationary state, the class of equivalence of eigenvectors $\psi$ of $H$, where the stationarity is characterized by the behavior $\psi(t)=\exp (-i E t) \psi \sim \psi$.

The parity $P$ and the time-reversal $T$ operators are defined respectively by

$$
P \psi(x)=\psi(-x) \text { and } T \psi(x)=\bar{\psi}(x) \text { for all } \psi \in \mathcal{H} .
$$

Since the operator $H$ is real, we have $T U_{t} T^{-1}=U_{-t}$, justifying the name of time reversal for the operator $T$.

Given a unitary operator $U$, we get the change of representation of the states $\psi \rightarrow U \psi$ for all $\psi \in \mathscr{H}$. In this representation the Hamiltonian becomes the unitarily equivalent Hamiltonian $H_{U}=U H U^{-1}$, with the same spectrum. In the case of the Fourier transform, $\mathscr{F}=U$, we pass from the position to the momentum representation. For the continuation of the resonances in the complex plane of the parameter, we use generalized changes of representation (see Section 1d of the sequel).

In the case of the hydrogen atom we are in $3 \mathrm{D}(d=3)$ :

$$
H_{0}=p^{2}+V(x), \quad x \in \mathbb{R}^{3}, p^{2}=-\nabla^{2}, V(x)=-K /|x|,
$$

where $K>0$ is fixed and

$$
D\left(H_{0}\right)=D\left(p^{2}\right) \subset \mathscr{H}=L^{2}\left(\mathbb{R}^{3}\right) .
$$

There are infinite eigenvalues of $H_{0}$, (energy) levels $E_{n}<0$, where the eigenfunctions are the stationary states $\psi_{n}$, since $\psi_{n}(t)=\exp \left(-i E_{n} t\right) \psi_{n} \sim \psi_{n}$. The absolute continuous spectrum $R_{+}$is related to the range of the mean energies $((\psi, H \psi)=E \geq 0)$ of the unstable (scattering) states $\psi \in D(H)$.

Now we consider the hydrogen Stark effect, with a perturbed hydrogen Hamiltonian

$$
H_{\epsilon}=H_{0}+\epsilon x_{1}, \quad \epsilon>0 .
$$

In this case there are resonances, complex numbers $E_{n}(\epsilon)$, such that $E_{n}(\epsilon) \rightarrow E_{n}$ as $\epsilon \rightarrow 0$. As introduced above, the corresponding states $\psi_{n}(\epsilon) \notin \mathscr{H}$, if suitably cut at infinity, become metastable states. This fact was proven by the complex scaling of the variables [Aguilar and Combes 1971] $x \rightarrow \lambda x$ and $p \rightarrow(1 / \lambda) p, \lambda \notin \mathbb{R}$ and $\Im \ln (\lambda)>0$, getting the non-s.a. operators

$$
H_{\epsilon}(\lambda)=\lambda^{-2}\left(p^{2}-\lambda(K /|x|)+\epsilon \lambda^{3} x_{1}\right) \quad \text { and } \quad D\left(H_{\epsilon}\right)=D\left(p^{2}\right) \cap D\left(x_{1}\right),
$$

with complex eigenvalues $E_{n}(\epsilon)$, invariant for $0<\Im \ln (\lambda)<\pi / 6$, representing the resonances of the Stark effect [Graffi and Grecchi 1978; Herbst and Simon 1978]. Notice that, by the use of the parabolic coordinates, the Stark effect in hydrogen can be decomposed into two anharmonic oscillators, one stable and the other one unstable. The perturbation series in $\epsilon$ of the resonances are divergent but summable 
by the distributional Borel method [Caliceti et al. 1986] to the real part of the resonances. Also the mean life of the metastable states is given by the same method.

\section{Some solvable models}

As basic solvable models, we consider the linear and the quadratic potentials.

We start with the Hamiltonian with linear potential

$$
H_{1}(\lambda)=\lambda^{-2}\left(p^{2}+\lambda^{3} x\right), \quad \lambda \neq 0, \Im \ln (\lambda)=\theta .
$$

In the case of $\lambda=1$, we have the self-adjoint Hamiltonian $H_{1}(1)$ with continuous spectrum $\sigma_{c}=\mathbb{R}$ [Kato 1966]. The resonances are given by the eigenvalues of the operators $H_{1}(\lambda)$ for $0<\theta<\pi / 3$ and domain $D\left(H_{1}(\lambda)\right)=D\left(p^{2}\right) \cap D(x)$. In particular, for $\theta=\pi / 6$, we have $H_{1}(\exp (i \pi / 6))=\exp (-i \pi / 3) O_{1}$ where

$$
O_{1}=p^{2}+i x
$$

is a PT operator with discrete spectrum [Herbst and Simon 1978]. Actually, because of the invariance of the spectrum to any translation $x \rightarrow x+\epsilon, \epsilon \in \mathbb{R}$, an eigenvalue $E_{n}$ should satisfy the impossible condition that there exists $m=m(n, \epsilon)$ such that $E_{n}=E_{m}+i \epsilon$. Thus, there are no resonances in this problem $H_{1}(1)$, as can be understood by the absence of wells or traps of the potential.

The other solvable Hamiltonians are

$$
H_{2}^{ \pm}(\lambda)=\lambda^{-2}\left(p^{2} \pm \lambda^{4} x^{2}\right), \quad \lambda \neq 0 .
$$

In particular,

$$
H_{2}^{+}(1):=O_{2}=p^{2}+x^{2}
$$

is the harmonic oscillator with simple levels $E_{n}=2 n+1, n \in \mathbb{N}$, and states $\psi_{n}(x)$. For $E=E_{n}$, the interval $[-\sqrt{E}, \sqrt{E}]$ is the internal Stokes line [Giller 2011] (oscillatory range) where all the zeros (nodes) of $\psi_{n}(x)$ lie.

In the case of $H_{2}^{-}(1)=p^{2}-x^{2}$, we have the repulsive harmonic oscillator with continuous spectrum $\sigma_{c}=\mathbb{R}$. The eigenvalues $E_{n}(\lambda)$ of the operator

$$
H_{2}^{-}(\lambda)=\lambda^{-2}\left(p^{2}-\lambda^{4} x^{2}\right), \quad \lambda \neq 0,
$$

are independent of $\lambda$ for $0<\Im \ln (\lambda)<\pi / 2$. Moreover, the eigenfunctions $\psi_{n}(\lambda, z)$, extended as entire functions in $z$, are simple dilations [Aguilar and Combes 1971] of $\psi_{n}(1, z): \psi_{n}(\lambda, z)=\lambda^{1 / 2} \psi_{n}(1, \lambda z)$. In particular, the spectrum of $H_{2}^{-}(\sqrt{i})=$ $-i\left(p^{2}+x^{2}\right)$ is the set of the top resonances of $H_{2}^{-}(1)$ :

$$
\sigma_{d}=\{-i(2 n+1)\}_{n \in \mathbb{N}} \text {. }
$$


Associated with these resonances are metastable states trapped about the top of the potential at $x=0$. Remark that the top of the potential is an unstable equilibrium point in classical mechanics.

Translations in the complex plane $x \rightarrow x+\alpha$ allow one to extend the family of operators. In particular, for $\lambda=1, \alpha=i$,

$$
H_{2}^{+}(1, i)=p^{2}+(x+i)^{2}=p^{2}+x^{2}+2 i x-1 \sim H_{2}^{+}(1)
$$

is a nontrivial PT operator with real levels $E_{n}=2 n+1$.

Now we consider some nonsolvable oscillators.

\section{The quartic oscillator}

The quartic oscillator is the first of the nonsolvable oscillators to be studied rigorously. It is defined by the family of Hamiltonians

$$
H_{1}(\beta)=p^{2}+V(x), \quad V(x)=x^{2}+\beta x^{4}, x \in \mathbb{R}, p^{2}=-\frac{d^{2}}{d x^{2}},
$$

where $\beta$ is in the cut plane

$$
\mathbb{C}_{c}=\{z \in \mathbb{C}: z \neq 0, z=|z| \exp (i \theta),|\theta|<\pi\},
$$

with domain $D\left(H_{1}\right)=D\left(p^{2}\right) \cap D\left(x^{4}\right)$.

For $\beta$ positive, $H_{1}$ is self-adjoint and the potential has a single well on the real axis. Actually, on the real axis the force $-V^{\prime}(x)=-2 x\left(1+4 \beta x^{2}\right)$ vanishes only at the origin.

For the corresponding levels $E_{n}(\beta)$, the Stieltjes or Padé summability of the perturbation series was proven in [Loeffel et al. 1969]. The main technique used was the Loeffel-Martin method of control of the zeros of the states in the complex plane. Later, the Borel summability of the perturbation series was proven in [Graffi et al. 1970]. Analytic continuations of the levels $E_{n}(\beta)$ beyond $\mathbb{C}_{c}$ suggested the existence of crossings (the Bender-Wu singularities [1969]).

We consider also the two-dimensional quartic oscillator by the reduction of the Hamiltonian to the radial operators:

$$
H_{2}(\beta, j)=p_{r}^{2}+\frac{j^{2}-1}{4 r^{2}}+r^{2}+\beta r^{4}, \quad r \in \mathbb{R}_{+}, p_{r}^{2}=-\frac{d^{2}}{d r^{2}},
$$

for $\beta \in \mathbb{C}_{c}$ and $j \geq 0$. The level $E_{n}(\beta, j)$, at the limit of the rim of the cut defined by $\arg (\beta)=\pi^{-},|\beta|=b>0$, is the resonance in the sense of the boundary conditions of Gamow [Caliceti and Maioli 1983] of the formal Hamiltonian $H_{2}(\beta, j)$ at $\beta=-b$. Since the hydrogen Stark effect can be decomposed by the parabolic coordinates in two operators of this kind, the existence of the resonances of the hydrogen Stark effect was also proven [Graffi and Grecchi 1978]. Moreover, 
the Borel distributional summability of the perturbation series of the same Stark resonances was proven in [Caliceti et al. 1993].

Of great interest is the double-well quartic oscillator defined by the families of self-adjoint Hamiltonians

$$
Q(\beta, j)=p^{2}+x^{2}(\beta x-1)^{2}-j\left(\beta x-\frac{1}{2}\right), \quad x \in \mathbb{R}, \beta \in \mathbb{R}_{+},
$$

for a fixed $j \in \mathbb{R}$. In particular, for $j=0$ we have the case of a symmetric double well. The states are of even or odd parity, and the levels are classified in doublets of opposite parity with a splitting of exponential order. The perturbation series are the same for both levels of a doublet so that the distributional sum of the series cannot give both the levels and in fact neither. In [Caliceti et al. 1988; 1996] a sequence of approximants to the levels up to the first exponential order was given by the distributional Borel sum [Caliceti et al. 1986] of the perturbation series.

\section{PT operators: a short story}

For the study of the resonances in the case of analytic potential, the introduction of non-self-adjoint Hamiltonians [Aguilar and Combes 1971] is useful. Sometimes the resonance Hamiltonian can be put in $P T$-symmetric form [Herbst and Simon 1978]. A PT operator is a real operator in the momentum representation, and its spectrum can be real.

I recall that some numerical investigations by Zinn-Justin suggested the coincidence of the perturbation series of the levels of two different problems: the unstable, rotationally symmetric, anharmonic oscillator in 2D and the double-well oscillator. Since usually a coincidence is not causal, the people of the scientific world looked for a stronger fact: the isospectrality of two Hamiltonian families. Many attempts were made without a full success. Eventually Buslaev and Grecchi [1993] proved the isospectrality of the self-adjoint double-well Hamiltonian $Q(\beta, j)$ of (6) and the radial reduction of the unstable anharmonic operator defined by a complex translation on the full axis as a PT operator:

$$
H_{\epsilon}(\beta, j)=\frac{1}{2}\left(p_{r}^{2}+\frac{j^{2}-1}{4 r_{\epsilon}^{2}}+r_{\epsilon}^{2}\right)-\beta^{2} r_{\epsilon}^{4}, \quad r \in \mathbb{R},
$$

where $p_{r}=-i \partial_{r}$ and $r_{\epsilon}=r-i \epsilon$, for an $\epsilon>0$. By some transformations on the variable and on the wave function, and also by the Fourier transform, it was proven that the two operators are isospectral, or equivalent in our terminology (the notion of equivalence in this case is not standard but was shared by others).

Scaling the variable, we also proved the isospectrality of the PT Hamiltonian

$$
O_{4}(\epsilon, \alpha, j)=\frac{1}{2}\left(p_{r}^{2}+\frac{j^{2}-1}{4 r_{\epsilon}^{2}}+\alpha r_{\epsilon}^{2}\right)-r_{\epsilon}^{4}, \quad r \in \mathbb{R}, r_{\epsilon}=r-i \epsilon,
$$


for an $\epsilon>0$, and the double-well one,

$$
Q(\alpha, j)=p^{2}+\left(x^{2}-\frac{1}{4} \alpha^{2}\right)^{2}-j x
$$

self-adjoint for $\alpha$ and $j$ real. As a consequence, we have proven the realness of the spectrum of families of PT Hamiltonians.

Our proofs are rigorous and obviously independent of the Zinn-Justin conjecture. For people interested in the paper [Benassi and Grecchi 1980], let me say that it is synthetic and very precise in the operatorial implementation of the analytic transforms.

Later, Bender and Boettcher [1998] discussed and Shin [2002] proved the realness of the spectrum of a class $O_{N}$ of $P T$-symmetric operators, containing our (7) only in the case of $j=1$.

\section{The cubic single-well Hamiltonian}

The cubic resonance problem is one of the simplest, not explicitly solvable ones in quantum mechanics [Caliceti et al. 1980; Grecchi and Martinez 2013; Grecchi et al. 2009; 2010; Caliceti 2000]. We consider the single (real) well Hamiltonian

$$
H(\beta)=p^{2}+x^{2}+i \sqrt{\beta} x^{3}, \quad \beta \in \mathbb{C}_{c} .
$$

We define nodes to be the $n$ zeros of state $\psi_{n}(\beta)$ stable at the unperturbed limit $\beta \rightarrow 0$.

Remark. The stability of the nodes at $\beta=0$ is equivalent to the stability of the levels because of the Cauchy theorem.

Also our results on the analyticity of the levels for large $|\beta|$ depend on the stability and isolation of the nodes.

(a) The first thing to prove is the absence of the essential spectrum. Thus, we prove the compactness of the resolvent.

Let us consider the Hamiltonian $H(\beta)$, for any fixed $\beta \in \mathbb{C}_{c}$. For any fixed $E \in \mathbb{C}$, the semiclassical behavior of the fundamental states [Sibuya 1975] implies that the Green's function $G(x, y)$ behaves as $|G(x, y)| \sim|x y|^{-3 / 4}$ for both $|x|,|y| \rightarrow \infty$ and is exponentially small if only one of the coordinates $(x, y)$ diverges, so that is in $L^{2}\left(\mathbb{R}^{2}\right)$. The corresponding integral operator, the inverse of $H(\beta)$, is HilbertSchmidt.

(b) The second thing to prove is the isolation of the nodes or the confinement and the stability of the nodes for the parameter on the cut plane.

(b1) The first part of the proof regards the existence of a no-vanishing zone [Delabaere and Trinh 2000]. 
The proof is based on the method of Loeffel and Martin [Loeffel et al. 1969] for the confinement of the zeros (9).

We consider the stripe of the complex plane of the variable $\beta$

$$
A(\beta)=\left\{z=x+i y \in \mathbb{C}: x, y \in \mathbb{R}, 0 \leq y \leq \frac{2}{3 \sqrt{b}} \cos \frac{\theta}{2}\right\},
$$

where $\beta=b \exp (i \theta)$.

Proposition. For all $\beta \in \Omega$, the eigenfunction $\psi_{\beta}$ does not admit any zeros on the stripe $A(\beta)$.

Let $V_{\beta}(z)=z^{2}+i \sqrt{\beta} z^{3}, z=x+i y$ [Loeffel et al. 1969]. Then

$$
\begin{aligned}
\Im \psi_{\beta}^{\prime}(x+i y) \overline{\psi_{\beta}(x+i y)} & =\int_{-\infty}^{x} \Im\left(V_{\beta}(r+i y)-E(\beta)\right)\left|\psi_{\beta}(r+i y)\right|^{2} d r \\
& =-\int_{x}^{\infty} \Im\left(V_{\beta}(r+i y)-E(\beta)\right)\left|\psi_{\beta}(r+i y)\right|^{2} d r
\end{aligned}
$$

It is enough to prove that the integrand changes sign at most once in $\mathbb{R}$ for any $z \in A(\beta)$. In this case at least one, and thus both, of the integrals in (9) are nonzero. Since $\left|\psi_{\beta}(z)\right|^{2} \geq 0$ and positive almost everywhere, we look for the monotonicity of the factor of the integrand

$$
\Im\left(V_{\beta}(r+i y)-E(\beta)\right)=2 r y+r^{3} \sqrt{b} c-3 r^{2} y \sqrt{b}-3 r y^{2} c-\Im(E(\beta)),
$$

where $c=\cos (\theta / 2)$ and $s=\sin (\theta / 2)$, or the nonvanishing of the derivative

$$
\begin{aligned}
\frac{d}{d r} \Im\left(V_{\beta}(r+i y)\right) & =3 \sqrt{b} c r^{2}-6 \sqrt{b} y s r+2 y-3 y^{2} \sqrt{b} c \\
& =3 \sqrt{b} c\left(r^{2}-2 y r \tan +\frac{2 y-3 y^{2} \sqrt{b} c}{3 \sqrt{b} c}\right),
\end{aligned}
$$

where $c=\cos (\theta / 2), s=\sin (\theta / 2)$ and $\tan =\tan (\theta / 2)$. The reduced discriminant is

$$
\Delta=9 y b\left(y-\frac{2}{3 \sqrt{b}} \cos \frac{\theta}{2}\right) \text {. }
$$

(b2) The second part of the proof regards the absence of zeros far from the origin in a large sector.

The eigenfunctions are $L^{2}$ on the real axis so that they are vanishing at infinity in two Stokes sectors

$$
S_{ \pm}(\beta)=\left\{x \in \mathbb{C}:\left|\arg (i x)+\frac{\theta}{10} \mp \frac{2 \pi}{5}\right|<\frac{\pi}{5}\right\} .
$$


This means that [Sibuya 1975] they are free of zeros (for large $|x|$ ) on two extended sectors

$$
S^{ \pm}(\beta)=\left\{x \in \mathbb{C}:\left|\arg (i x)+\frac{\theta}{10} \mp \frac{2 \pi}{5}\right|<\frac{3 \pi}{5}\right\} .
$$

Thus, the sequence of zeros $N_{j}$ of $\psi_{n}(x, \beta)$ have the asymptotic phase $\pi / 2+\theta / 10$, as $\left|N_{j}\right| \rightarrow \infty$.

\section{(c) The third thing to prove is the absence of nonperturbative levels.}

It is worth recalling this proof since it looks difficult but needs only a careful analysis.

Let $E(\beta)$ be an eigenvalue defined as a multiple-valued analytic function on $\mathbb{C}_{c}$, with normalized eigenfunction $\psi_{\beta}$, and let $E_{n}(\beta)$ be a perturbative eigenvalue for $b$ small.

Proposition. Let $\widetilde{E}(\beta)$ be a determination of $E(\beta)$; then on a disk $|\beta|<b_{0}, \beta \in \mathbb{C}_{c}$, $\widetilde{E}(\beta)=E_{n}(\beta)$ for $a b_{0}>0$ and $n \in \mathbb{N}$.

Remark. A consequence of this proposition is that the branch points of $\widetilde{E}(\beta)$ in $\mathbb{C}_{c}$ do not accumulate at 0 .

Proof. Let $\gamma$ be a simple path in $\mathbb{C}_{c} \cup\{0\}$ such that $\widetilde{E}(\beta)$ is analytic in a neighborhood of $\gamma$ in $\mathbb{C}_{c}$, bypassing the possible sequence of positive branch points on $\gamma$, with $\beta_{k} \in \gamma$ and $\beta_{k} \rightarrow 0$ as $k \rightarrow \infty$.

Let us distinguish two cases.

Case 1. The sequence $\left(\left|\widetilde{E}\left(\beta_{k}\right)\right|\right)_{k}$ is bounded. In this case, we have a subsequence converging to a limit $\widetilde{E}\left(\beta_{k}\right) \rightarrow E_{n}$, where $E_{n}=E_{n}(0)$ is an eigenvalue of the unperturbed Hamiltonian because of the stability. Moreover, for the stability and the perturbation theory, we have $\widetilde{E}(\beta)=E_{n}(\beta)$ for $\beta \in \gamma,|\beta|<b_{0}$, for a $b_{0}>0$.

Case 2. $\left|\widetilde{E}\left(\beta_{k}\right)\right| \rightarrow \infty$ as $k \rightarrow \infty$.

Let us split $B:=\left(\beta_{k}\right)_{k}$ into

$$
B_{ \pm}=\left\{\beta \in B: \pm|\widetilde{E}(\beta)| \leq \pm \beta^{-1}\right\} .
$$

(2a) When $\beta \in B_{-}$we make the change of variable $x=\lambda y$, with $\lambda:=\sqrt{|\widetilde{E}(\beta)|}$, and we set

$$
\phi(y)=\sqrt{\lambda} \psi_{\beta}(\lambda y), \quad h:=\frac{1}{\lambda^{2}}, \quad b^{\prime}=\lambda^{2} \sqrt{\beta} \in(0,1] \quad \text { and } \quad u:=\widetilde{E}(\beta) /|\widetilde{E}(\beta)|
$$

so that we have

$$
\left(H_{h}\left(b^{\prime}\right)-u\right) \phi(y)=-h^{2} \phi^{\prime \prime}(y)+\left(y^{2}+i \sqrt{b^{\prime}} y^{3}-u\right) \phi(y)=0,
$$

and we can use the complex semiclassical method for $\alpha$ and $\hbar$ small [Voros 1994]. Since $b^{\prime}>0$, the numerical range of $H_{h}\left(b^{\prime}\right)$ and $u$ are in the half-plane $\Re z \geq 1$. Thus, $\arg (u)<\pi / 2$ and two turning points $y_{ \pm}\left(b^{\prime}, u\right)$ of (10) are located in the 
half-plane $\Im y \leq 1 / \sqrt{2}$, while the third one is in $\Im y \geq 1$. In this case there are no

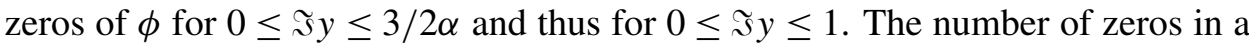
disk of fixed radius $\left|y_{+}\left(b^{\prime}, u\right)-y\right| \leq \delta$ diverges as $O(1 / h)$ in contradiction with the finite constant number of the nodes.

(2b) When $\beta \in B_{+}$, with $\lambda:=(|\widetilde{E}(\beta)| / \sqrt{\beta})^{1 / 3}$, we make the change of variable $x=\lambda y$, and we define the new function

$$
\phi(y)=\sqrt{\lambda} \psi_{\beta}(\lambda y)
$$

to be the solution of the equation

$$
-h^{2} \phi^{\prime \prime}(y)+\left(\alpha y^{2}+i y^{3}-u\right) \phi(y)=0,
$$

with $u=E(\beta) /|E(\beta)|, h=1 / \lambda \sqrt{|E(\beta)|} \rightarrow 0$ as $\beta \rightarrow 0$ and $0<\alpha=(\beta|\widetilde{E}(\beta)|)^{-1 / 3} \leq$ 1 as $\beta \rightarrow 0$.

In this case, where $\beta \in B_{+}$, we prove the same contradiction as in the case $B_{-}$. In particular, if $\alpha \rightarrow 0$ both $\Im y_{ \pm}$become negative as in the $\beta \rightarrow \infty$ case.

\section{(d) Now we prove that the levels are Stieltjes functions.}

The proof that

$$
f(\beta):=-\frac{E_{n}(\beta)-E_{n}(0)}{\beta}, \quad n \in \mathbb{N},
$$

is a Stieltjes function is based on its analyticity and boundedness on $\mathbb{C}_{c}$ and the definite sign of the imaginary part of $\Im E_{n}(-b-i 0) \leq 0$. The sign of $\Im E_{n}(-b-i 0)$ comes from the Gamow condition of the corresponding state $\phi(x)$ at $-\infty$. The state $\phi(x)$ and $\phi^{\prime}(x)$ have no zeros on the real axis. The level is a generalized resonance, or a limit of ordinary resonances, and its imaginary part, with changed sign, is related to the inverse mean life of the associated metastable states. The resonance eigenfunction satisfies the Gamow condition for $-x>0$ large, and by the semiclassical behavior [Sibuya 1975], we have

$$
\begin{gathered}
\hbar \frac{\phi^{\prime}(x)}{\phi(x) \sqrt{E-V(x)}} \rightarrow-i, \quad \sqrt{E-V(x)}|\phi(x)|^{2} \rightarrow|c|^{2}>0, \\
-\Im E=-\frac{\hbar^{2}}{\int_{x}^{\infty}|\phi(y)|^{2} d y} \Im\left(\frac{\phi^{\prime}(x)}{\phi(x)}|\phi(x)|^{2}\right) \rightarrow \hbar|c|^{2} .
\end{gathered}
$$

\section{The cubic $P T$-symmetric double well}

The single-well cubic oscillator discussed above can be analytically continued by generalized changes of representation [Aguilar and Combes 1971; Graffi and Grecchi 1978], obtaining the family of PT Hamiltonians

$$
H_{\hbar}=\hbar^{2} p^{2}+V(x), \quad V(x)=i\left(x^{3}-x\right), \hbar>0 .
$$


Since $V^{\prime}(x)=0$ at the two points $x_{ \pm}= \pm 1 / \sqrt{3} \in \mathbb{R}$ (wells), we have a double-well PT Hamiltonian, whose states possibly localize about one of the wells (localization) or about both (delocalization or bilocalization). The transition from one to the other kind of state is singular and happens at the level crossing.

Also in the case of the unstable double-well Stark effect, varying the field parameter, there is a double level crossing and the states are localized for large negative and large positive parameter, but not for small parameter [Grecchi et al. 1996].

The existence of the level crossings is the main issue of the research on this model. The levels at the crossings are near a critical energy, usually the top of the internal barrier. In our PT case the internal barrier doesn't exist and the critical energy is a singularity of the Stokes graph [Giller 2011; Delabaere and Trinh 2000].

The localization is a typical semiclassical effect, since the classical particles, at low energy, are localized in one of the wells.

Actually, in usual quantum mechanics with a family of self-adjoint Hamiltonians for all real parameters, the delocalization of the states is stable and the level crossings are forbidden [Kato 1966; Reed and Simon 1975; 1978]. Also in the case of single-well PT Hamiltonians [Shin 2002; Caliceti 2000; Grecchi and Martinez 2013; Grecchi et al. 2009; 2010] the states are always localized about the well and the crossings are absent.

The semiclassical theory provides good qualitative and quantitative results on the levels $E_{n}(\hbar)$ for lower parameter $\hbar$ up to the crossing value [Bender and $\mathrm{Wu}$ 1969; Benassi and Grecchi 1980; Harrell and Simon 1980; Alvarez 1995]. The complex semiclassical method [Voros 1994; Delabaere et al. 1997] has extended the results to larger, but not very large, values of the parameter [Delabaere and Trinh 2000; Delabaere and Pham 1997]. Recently, rigorous results were also obtained in [Eremenko and Gabrielov 2011] by different techniques. Such approaches are useful and complementary to our rigorous treatment based on the nodal analysis [Giachetti and Grecchi 2015; 2016a; 2016b]. The nodal analysis is found also in other papers [Grecchi and Martinez 2013; Grecchi et al. 2009; 2010; Eremenko et al. 2008a; 2008b; Shanley 1988a; 1988b; Eremenko and Gabrielov 2009].

As proven above, all the states $\tilde{\psi}_{n}(\beta)$, with corresponding levels $\widetilde{E}_{n}(\beta)$, are perturbative and their subscripts $n$ are the numbers of their zeros stable at $\beta=0$ (nodes). The result is relevant to our present context, as we may observe that the Hamiltonians (12) and (8) are related by a generalized change of representation (with a definite law of transformation of the levels). Indeed, making the unitary translations $x=x_{ \pm}+y$, we obtain

$$
H_{\hbar}^{ \pm}=\hbar^{2} p^{2}+i\left(y^{3} \pm \sqrt{3} y^{2}\right) \mp i E_{0}, \quad E_{0}=\frac{2}{3 \sqrt{3}} .
$$


In order to connect $H_{\hbar}^{ \pm}$with $H(\beta)$ in the sectors $\pi<\mp \arg (\beta)<3 \pi / 2$, respectively, we consider the analytic dilations [Aguilar and Combes 1971]

$$
y=\lambda^{ \pm}(\hbar) z, \quad \lambda^{ \pm}(\hbar)=3^{-1 / 8} \sqrt{\hbar} \exp (\mp i \pi / 8),
$$

conserving the spectrum. Letting $c^{ \pm}=3^{1 / 4} \sqrt{ \pm i}$ we find from (8)

$$
H\left(\beta^{ \pm}(\hbar)\right) \sim\left(1 / \hbar c^{ \pm}\right) H_{\hbar}^{ \pm} \pm i E_{0} \quad \text { and } \quad E_{n}\left(\beta^{ \pm}(\hbar)\right)=\left(1 / \hbar c^{ \pm}\right) E_{n}^{ \pm}(\hbar) \pm i E_{0}
$$

where

$$
\beta^{ \pm}(\hbar)=\exp (\mp i 5 \pi / 4) 3^{-5 / 4} \hbar
$$

Notice that

$$
E_{n}^{ \pm}(\hbar)=\hbar^{6 / 5} \widehat{E}_{n}\left(\alpha^{\mp}\right), \quad n \in \mathbb{N}, \quad \text { and } \quad \alpha^{ \pm}=\exp ( \pm i \pi) \hbar^{-4 / 5}, \quad \hbar>0 .
$$

We see that levels $E_{n}^{ \pm}(\hbar)$ are complex conjugates and are different for small $\hbar>0$, since their semiclassical behaviors are

$$
E_{n}^{ \pm}(\hbar)=\mp i E_{0}+\sqrt{ \pm i} \sqrt[4]{3}(2 n+1) \hbar+O\left(\hbar^{2}\right) \in \mathbb{C}_{\mp}=\{z \in \mathbb{C}: \mp \Im \mathfrak{I} z>0\} .
$$

The zeros of $\psi_{n}^{ \pm}(\hbar, z)$ for large $|z|$ are contained respectively in

$$
\mathbb{C}^{ \pm}=\{z \in \mathbb{C}: \pm \Re z>0\} .
$$

The large- $\hbar$ behavior of the levels $E_{m}(\hbar)$ is studied by using a different representation with the Hamiltonians

$$
O_{3}(\alpha)=p^{2}+W(\alpha, x), \quad W(\alpha, x)=i\left(x^{3}+\alpha x\right), \alpha \in \mathbb{C} .
$$

The eigenvalue $\widehat{E}_{m}(\alpha)$ of $O_{3}(\alpha)$ is holomorphic in a neighborhood of the origin. Thus, for large positive $\hbar$ the levels $E_{m}(\hbar)$ are defined by

$$
\hbar^{-6 / 5} E_{m}(\hbar)=\widehat{E}_{m}(\alpha), \quad \alpha=-\hbar^{-4 / 5} \leq 0,
$$

and they are connected with the eigenvalues of $H(\beta)$ by the relation

$$
\widehat{E}_{m}(\alpha(\beta))=\beta^{-1 / 5} \widetilde{E}_{m}(\beta)+\frac{2 \beta^{-1 / 5}}{27 \beta}, \quad \alpha(\beta)=\frac{1}{3 \beta^{4 / 5}} \geq 0,
$$

at $\alpha=0$. The level functions $\widehat{E}_{m}(\alpha)$ are real-analytic for $\alpha \in \mathbb{R},|\alpha|$ small. Thus, all such levels $E_{m}(\hbar)$ are given by analytic continuations of the perturbative levels $\widetilde{E}_{m}(\beta)$ through $(21)$ and $(22)$, and are extensible as many-valued functions (taking different names), to the sector

$$
\mathbb{C}^{0}=\{z \in \mathbb{C}: z \neq 0,|\arg (z)|<\pi / 4\}
$$

of the $\hbar$ complex plane. The corresponding states $\psi_{m}(\hbar)$ are delocalized (or bilocalized about both wells). 
The crossing selection rule is given in simple terms: the two positive levels $E_{2 n+(1 / 2) \pm(1 / 2)}(\hbar)$ with delocalized states $\psi_{2 n+(1 / 2) \pm(1 / 2)}(\hbar), \hbar>\hbar_{n}$, cross at $\hbar_{n}>0$ and become the two complex levels $E_{n}^{ \pm}(\hbar)$ with localized states $\psi_{n}^{ \pm}(\hbar)$ for $\hbar<\hbar_{n}$. This selection rule completely describes the full crossing process.

The two levels $E_{n}^{ \pm}(\hbar)$ of (15) and (17) are complex-conjugated, and the two states $\psi_{n}^{ \pm}(\hbar)$ are $P T$-conjugated and concentrated about the wells $x_{ \pm}$, respectively.

This process is possible because of the instability of the delocalization of both the states $\psi_{2 n+(1 / 2) \pm(1 / 2)}(\hbar)$ for decreasing $\hbar>0$.

Conclusion. The nodes of the states $\psi_{2 n+(1 \pm 1) / 2}(\hbar)$ for large $\hbar>0$, with positive levels $E_{2 n+(1 \pm 1) / 2}(\hbar)$, are in the half-plane $\mathbb{C}_{-}$. Only the state $\psi_{2 n+1}(\hbar)$ has an unstable imaginary node. The number of nonimaginary nodes of both the states $\psi_{2 n+(1 \pm 1) / 2}(\hbar)$ are $2 n, n$ of them in $\mathbb{C}^{ \pm}$.

The nodes of the states $\psi_{n}^{ \pm}(\hbar)$ for $h b<\hbar_{n}$ are in $\mathbb{C}^{ \pm}$, respectively.

\section{A simple model for the racemization}

Many years ago Friedrich Hund proposed a model [Dennison and Uhlenbeck 1932] for the ammonia molecule $\mathrm{NH}_{3}$. Supposing the hydrogen atoms lie in a plane, he considered the motion of the nitrogen atom along an orthogonal axis. The forces acting on the $\mathrm{N}$ atom should correspond to a double-well potential because of the symmetry and the typical interatomic forces. In this quantum model the $\mathrm{N}$ atom state is delocalized or localized about both the wells. Actually, we know that the molecule in a dense gas has a structure and the $\mathrm{N}$ atom is localized. It is possible to represent this effect by a nonlinear model [Grecchi and Martinez 1995].

A molecule with a structure is a metastable state of its system [Woolley 1982]. Here we consider a linear model with a PT Hamiltonian in order to define the resonant state related to the metastable one. The delocalized state of the $\mathrm{N}$ atom for low density of the gas can be represented by our $P T$ states $\psi_{2 n+(1 \pm 1) / 2}(\hbar)$ of (12) for large $\hbar>0$. The splitting $\Delta E=E_{2 n+1}(\hbar)-E_{2 n}(\hbar)$ gives the pulsation of the inversion line $\omega=\Delta E / \hbar$ and the beating effect. The dynamics of the packet

$$
\psi=C\left(\psi_{2 n+1}(\hbar)+\psi_{2 n}(\hbar)\right),
$$

is

$$
\psi(t)=C(t) \exp (-i E t / \hbar)\left(\psi_{2 n+1}(\hbar)+\exp (-i \omega t) \psi_{2 n}(\hbar)\right),
$$

where $E=E_{2 n+1}(\hbar)$, showing the beating effect with the pulsation of the so-called inversion line.

For large density of the gas, we expect a localization of the nitrogen atom in one side of the plane of the hydrogen atoms, so that the structure of the molecule is pyramidal. If two of the hydrogen atoms are substituted by different isotopes $\mathrm{NH}_{3} \rightarrow$ NHDT, the pyramidal structure of the molecule gets a definite chirality. 
Actually, the model is not complete since there exists the racemization effect: a slow change of chirality.

In our model we can represent the state by one of the eigenfunctions $\psi(0)=$ $\psi_{n}^{ \pm}(\hbar), \hbar<\hbar_{n}$, localized about one of the wells $x_{ \pm}$, respectively. This state is stationary:

$$
\psi(t)=C(t) \exp (-i E t / \hbar) \psi(0), \quad E=E_{n}^{ \pm}(\hbar),
$$

where the coefficient $C(t)>0$ is given by the unitary normalization $\|\psi(t)\|=1$.

But it is also possible to represent the racemization.

The dynamics of a state is given by $\psi(t)=C(t) \exp \left(-i H_{\hbar} t\right) \psi(0)$, where $C(t)>$ 0 is given by the unitary normalization $\|\psi(t)\|=1$. Let us consider the $P T$ state as a wave packet at $t=0$,

$$
\psi(0)=C(0)\left(\psi_{n}^{-}(\hbar)+\psi_{n}^{+}(\hbar)\right)
$$

with unitary norm, for $\hbar>0$ small. This packet is $P T$-symmetric and is delocalized, or better, it is bilocalized about both the wells. The dynamics of the packet is

$$
\psi(t)=C(t)\left(\exp (-i \bar{E} t / \hbar) \psi_{n}^{-}(\hbar)+\exp (-i E t / \hbar) \psi_{n}^{+}(\hbar)\right),
$$

where $E=E_{n}^{+}(\hbar)$. Since $\Im E<0$, we have $|\psi(t)| \rightarrow\left|\psi_{n}^{\mp}(\hbar)\right|$ as $t \rightarrow \pm \infty$. Thus, we have a process of localization of the packet about the well $x_{\mp}$ in the limit $t \rightarrow \pm \infty$, respectively. The full process for $t$ going from $-\infty$ to $\infty$ is a slow change of localization of the $\mathrm{N}$ atom from a well to the other one. This corresponds to a change of chirality of the molecule or a racemization [Streitwieser and Heathcock 1985, p. 122-124].

In this case it is evident that the $P$ and $T$ symmetry of the process breaks, conserving the $P T$ symmetry. If $\hbar_{n}-\hbar$ is small enough, the time of the racemization process is much longer than the period of the beating effect.

I don't attempt to justify the model from a physical point of view. As a physical comment, I would say that, in general, time symmetry breaking is a consequence of space symmetry breaking. A rigid body is not a system in a stationary state since it breaks the space symmetry of the potential [Woolley 1982]. Thus, it breaks translational time symmetry.

\section{References}

[Aguilar and Combes 1971] J. Aguilar and J. M. Combes, "A class of analytic perturbations for one-body Schrödinger Hamiltonians”, Comm. Math. Phys. 22:4 (1971), 269-279.

[Alvarez 1995] G. Alvarez, "Bender-Wu branch points in the cubic oscillator", J. Phys. A 28:16 (1995), 4589-4598.

[Benassi and Grecchi 1980] L. Benassi and V. Grecchi, "Resonances in the Stark effect and strongly asymptotic approximants", J. Phys. B 13:5 (1980), 911-930. 
[Bender and Boettcher 1998] C. M. Bender and S. Boettcher, "Real spectra in non-Hermitian Hamiltonians having $\mathscr{P} \mathscr{T}$ symmetry", Phys. Rev. Lett. 80:24 (1998), 5243-5246.

[Bender and Wu 1969] C. M. Bender and T. T. Wu, "Anharmonic oscillator", Phys. Rev. (2) 184:5 (1969), 1231-1260.

[Buslaev and Grecchi 1993] V. Buslaev and V. Grecchi, "Equivalence of unstable anharmonic oscillators and double wells", J. Phys. A 26:20 (1993), 5541-5549.

[Caliceti 2000] E. Caliceti, "Distributional Borel summability of odd anharmonic oscillators", $J$. Phys. A 33:20 (2000), 3753-3770.

[Caliceti and Maioli 1983] E. Caliceti and M. Maioli, "Odd anharmonic oscillators and shape resonances”, Ann. Inst. H. Poincaré Sect. A (N.S.) 38:2 (1983), 175-186.

[Caliceti et al. 1980] E. Caliceti, S. Graffi, and M. Maioli, "Perturbation theory of odd anharmonic oscillators", Comm. Math. Phys. 75:1 (1980), 51-66.

[Caliceti et al. 1986] E. Caliceti, V. Grecchi, and M. Maioli, "The distributional Borel summability and the large coupling $\Phi^{4}$ lattice fields", Comm. Math. Phys. 104:1 (1986), 163-174.

[Caliceti et al. 1988] E. Caliceti, V. Grecchi, and M. Maioli, "Double wells: perturbation series summable to the eigenvalues and directly computable approximations", Comm. Math. Phys. 113:4 (1988), 625-648.

[Caliceti et al. 1993] E. Caliceti, V. Grecchi, and M. Maioli, "Stark resonances: asymptotics and distributional Borel sum", Comm. Math. Phys. 157:2 (1993), 347-357.

[Caliceti et al. 1996] E. Caliceti, V. Grecchi, and M. Maioli, "Double wells: Nevanlinna analyticity, distributional Borel sum and asymptotics", Comm. Math. Phys. 176:1 (1996), 1-22.

[Delabaere and Pham 1997] E. Delabaere and F. Pham, "Unfolding the quartic oscillator", Ann. Physics 261:2 (1997), 180-218.

[Delabaere and Trinh 2000] E. Delabaere and D. T. Trinh, "Spectral analysis of the complex cubic oscillator", J. Phys. A 33:48 (2000), 8771-8796.

[Delabaere et al. 1997] E. Delabaere, H. Dillinger, and F. Pham, "Exact semiclassical expansions for one-dimensional quantum oscillators", J. Math. Phys. 38:12 (1997), 6126-6184.

[Dennison and Uhlenbeck 1932] D. M. Dennison and G. E. Uhlenbeck, "The two-minima problem and the ammonia molecule", Phys. Rev. 41:3 (1932), 313-321.

[Eremenko and Gabrielov 2009] A. Eremenko and A. Gabrielov, "Analytic continuation of eigenvalues of a quartic oscillator", Comm. Math. Phys. 287:2 (2009), 431-457.

[Eremenko and Gabrielov 2011] A. Eremenko and A. Gabrielov, "Singular perturbation of polynomial potentials with applications to PT-symmetric families", Mosc. Math. J. 11:3 (2011), 473-503, 629-630.

[Eremenko et al. 2008a] A. Eremenko, A. Gabrielov, and B. Shapiro, "High energy eigenfunctions of one-dimensional Schrödinger operators with polynomial potentials", Comput. Methods Funct. Theory 8:2 (2008), 513-529.

[Eremenko et al. 2008b] A. Eremenko, A. Gabrielov, and B. Shapiro, "Zeros of eigenfunctions of some anharmonic oscillators", Ann. Inst. Fourier 58:2 (2008), 603-624.

[Giachetti and Grecchi 2011] R. Giachetti and V. Grecchi, " $P T$-symmetric operators and metastable states of the 1D relativistic oscillators", J. Phys. A 44:9 (2011), 095308.

[Giachetti and Grecchi 2015] R. Giachetti and V. Grecchi, "Localization of the states of a $P T$ symmetric double well”, Internat. J. Theoret. Phys. 54:11 (2015), 3889-3899.

[Giachetti and Grecchi 2016a] R. Giachetti and V. Grecchi, "Bender-Wu singularities", J. Math. Phys. 57:12 (2016), 122109. 
[Giachetti and Grecchi 2016b] R. Giachetti and V. Grecchi, "Level crossings in a $P T$-symmetric double well", J. Phys. A 49:10 (2016), 105202.

[Giller 2011] S. Giller, "The semiclassical small- $\hbar$ limit of loci of roots of subdominant solutions for polynomial potentials", J. Math. Phys. 52:6 (2011), 063514, 44.

[Graffi and Grecchi 1978] S. Graffi and V. Grecchi, "Resonances in Stark effect and perturbation theory", Comm. Math. Phys. 62:1 (1978), 83-96.

[Graffi et al. 1970] S. Graffi, V. Grecchi, and B. Simon, "Borel summability: application to the anharmonic oscillator", Phys. Lett. B 32:7 (1970), 631-634.

[Grandi 1710] G. Grandi, Quadratura circuli, et hyperbola per infinitas hyperbolas, et parabolas quadrabiles geometricè exhibita, et demonstrata, Francisci Bindi, Pisa, 1710.

[Grecchi and Martinez 1995] V. Grecchi and A. Martinez, "Non-linear Stark effect and molecular localization”, Comm. Math. Phys. 166:3 (1995), 533-548.

[Grecchi and Martinez 2013] V. Grecchi and A. Martinez, "The spectrum of the cubic oscillator", Comm. Math. Phys. 319:2 (2013), 479-500.

[Grecchi et al. 1996] V. Grecchi, A. Martinez, and A. Sacchetti, "Double well Stark effect: crossing and anticrossing of resonances", Asymptotic Anal. 13:4 (1996), 373-391.

[Grecchi et al. 2009] V. Grecchi, M. Maioli, and A. Martinez, "Padé summability of the cubic oscillator", J. Phys. A 42:42 (2009), 425208.

[Grecchi et al. 2010] V. Grecchi, M. Maioli, and A. Martinez, "The top resonances of the cubic oscillator", J. Phys. A 43:47 (2010), 474027.

[Hamilton 1834] W. R. Hamilton, "On a general method in dynamics, by which the study of the motions of all free systems of attracting or repelling points is reduced to the search and differentiation of one central relation, or characteristic function", Phil. Trans. R. Soc. Lond. 124 (1834), 247-308.

[Hamilton 1835] W. R. Hamilton, "On the application to dynamics of a general mathematical method previously applied to optics", pp. 513-518 in Report of the Fourth Meeting of the British Association for the Advancement of Science (Edinburgh, 1834), John Murray, London, 1835.

[Harrell and Simon 1980] E. Harrell and B. Simon, "The mathematical theory of resonances whose widths are exponentially small”, Duke Math. J. 47:4 (1980), 845-902.

[Herbst and Simon 1978] I. W. Herbst and B. Simon, "Stark effect revisited", Phys. Rev. Lett. 41:2 (1978), 67-69.

[Kato 1966] T. Kato, Perturbation theory for linear operators, Grundlehren math. Wissenschaften 132, Springer, Berlin, 1966.

[Loeffel et al. 1969] J. J. Loeffel, A. Martin, B. Simon, and A. S. Wightman, "Pade approximants and the anharmonic oscillator", Phys. Lett. B 30:9 (1969), 656-658.

[Martinez and Nédélec 2012] A. Martinez and L. Nédélec, "Optimal lower bound of the resonance widths for a Helmholtz tube-shaped resonator", J. Spectr. Theory 2:2 (2012), 203-223.

[Mengoli 1670] P. Mengoli, Speculationi di musica, Herede del Benacci, Bologna, 1670.

[Reed and Simon 1975] M. Reed and B. Simon, Methods of modern mathematical physics, II: Fourier analysis, self-adjointness, Academic, New York, 1975.

[Reed and Simon 1978] M. Reed and B. Simon, Methods of modern mathematical physics, IV: Analysis of operators, Academic, New York, 1978.

[Shanley 1988a] P. E. Shanley, "Nodal properties of the scaled quartic anharmonic oscillator", Ann. Physics 186:2 (1988), 325-354.

[Shanley 1988b] P. E. Shanley, "Spectral properties of the scaled quartic anharmonic oscillator", Ann. Physics 186:2 (1988), 292-324. 
[Shin 2002] K. C. Shin, "On the reality of the eigenvalues for a class of $\mathscr{P} \mathcal{T}$-symmetric oscillators", Comm. Math. Phys. 229:3 (2002), 543-564.

[Sibuya 1975] Y. Sibuya, "Distribution of zeros", Chapter 7, pp. 152-165 in Global theory of a second order linear ordinary differential equation with a polynomial coefficient, Math. Studies 18, North-Holland, Amsterdam, 1975.

[Streitwieser and Heathcock 1985] A. Streitwieser and C. H. Heathcock, Introduction to organic chemistry, 3rd ed., Macmillian, New York, 1985.

[Voros 1994] A. Voros, "Exact quantization condition for anharmonic oscillators (in one dimension)", J. Phys. A 27:13 (1994), 4653-4661.

[Woolley 1982] R. G. Woolley, "Natural optical activity and the molecular hypothesis", pp. 1-35 in Structures versus special properties, edited by M. J. Clarke et al., Struct. Bond. 52, Springer, Berlin, 1982.

Received 25 Mar 2016. Revised 29 Sep 2016. Accepted 31 Oct 2016.

VINCENZO GRECCHI: vincenzo.grecchi@unibo.it

Dipartimento di Matematica, Università di Bologna, I-40126 Bologna, Italy

and

Gruppo Nazionale di Fisica Matematica, Istituto Nazionale di Alta Matematica Francesco Severi, I-00185 Roma, Italy 
EDITORIAL BOARD

ANTONIO CARCATERRA

ERIC A. CARLEN

FRANCESCO DELL'ISOLA

RAFFAELE ESPOSITO

ALBERT FANNJIANG

Gilles A. FranCFORT

Pierangelo MARCATI

JEAN-JACQUES MARIGO

PETER A. MARKOWICH

MARTIN OSTOJA-STARZEWSKI

PIERRE SEPPECHER

DAVID J. STEIGMANN

PAUl STEINMANN

PierRe M. SuQueT

MANAGING EDITORS

MICOL AMAR

CORRADO LATTANZIO

ANGELA MADEO

MARTIN OSTOJA-STARZEWSKI

ADVISORY BOARD

ADNAN AKAY

Holm AltenBaCH

MICOL AMAR

HARM ASKES

TEODOR ATANACKOVIĆ

VICTOR BERDICHEVSKY

GUY BOUCHITTÉ

ANDREA BRAIDES

ROBERTO CAMASSA

MAURO CARFORE

ERIC DARVE

FELIX DARVE

ANNA DE MASI

GianPiEtro DEL Piero

EMMANUELE Di BENEDETTO

BERNOLD FIEDLER

IRENE M. GAMBA

DAVID Y. GAO

SERGEY GAVRILYUK

TIMOTHY J. HEALEY

DOMINIQUE JEULIN

ROGER E. KHAYAT

CORRADO LATTANZIO

ROBERT P. LIPTON

ANGELO LUONGO

ANGELA MADEO

JUAN J. MANFREDI

CARLO MARCHIORO

GÉRARD A. MAUGIN

ROBERTO NATALINI PATRIZIO NEFF

ANDREY PIATNITSKI

ERRICO PRESUTTI

MARIO PULVIRENTI

LUCIO RUSSO

Miguel A. F. SANJUAN

PATRICK SElVADURAI

ALEXANDER P. SEYRANIAN

MIROSLAV ŠILHAVÝ

GUIDO SWEERS

ANTOINETTE TORDESILLAS

LEV TRUSKINOVSKY

JUAN J. L. VELÁZQUEZ VINCENZO VESPRI ANGELO VULPIANI msp.org/memocs

Università di Roma "La Sapienza", Italia

Rutgers University, USA

(CO-CHAIR) Università di Roma "La Sapienza", Italia

(TREASURER) Università dell'Aquila, Italia

University of California at Davis, USA

(CO-CHAIR) Université Paris-Nord, France

Università dell'Aquila, Italy

École Polytechnique, France

DAMTP Cambridge, UK, and University of Vienna, Austria

(CHAIR MANAGING EDITOR) Univ. of Illinois at Urbana-Champaign, USA

Université du Sud Toulon-Var, France

University of California at Berkeley, USA

Universität Erlangen-Nürnberg, Germany

LMA CNRS Marseille, France

Università di Roma "La Sapienza", Italia

Università dell'Aquila, Italy

Université de Lyon-INSA (Institut National des Sciences Appliquées), France

(CHAIR MANAGING EDITOR) Univ. of Illinois at Urbana-Champaign, USA

Carnegie Mellon University, USA, and Bilkent University, Turkey

Otto-von-Guericke-Universität Magdeburg, Germany

Università di Roma "La Sapienza", Italia

University of Sheffield, UK

University of Novi Sad, Serbia

Wayne State University, USA

Université du Sud Toulon-Var, France

Università di Roma Tor Vergata, Italia

University of North Carolina at Chapel Hill, USA

Università di Pavia, Italia

Stanford University, USA

Institut Polytechnique de Grenoble, France

Università dell'Aquila, Italia

Università di Ferrara and International Research Center MEMOCS, Italia

Vanderbilt University, USA

Freie Universität Berlin, Germany

University of Texas at Austin, USA

Federation University and Australian National University, Australia

Université Aix-Marseille, France

Cornell University, USA

École des Mines, France

University of Western Ontario, Canada

Università dell' Aquila, Italy

Louisiana State University, USA

Università dell'Aquila, Italia

Université de Lyon-INSA (Institut National des Sciences Appliquées), France University of Pittsburgh, USA

Università di Roma "La Sapienza”, Italia

Université Paris VI, France

Istituto per le Applicazioni del Calcolo "M. Picone", Italy

Universität Duisburg-Essen, Germany

Narvik University College, Norway, Russia

Università di Roma Tor Vergata, Italy

Università di Roma "La Sapienza”, Italia

Università di Roma “Tor Vergata”, Italia

Universidad Rey Juan Carlos, Madrid, Spain

McGill University, Canada

Moscow State Lomonosov University, Russia

Academy of Sciences of the Czech Republic

Universität zu Köln, Germany

University of Melbourne, Australia

École Polytechnique, France

Bonn University, Germany

Università di Firenze, Italia

Università di Roma La Sapienza, Italia

MEMOCS (ISSN 2325-3444 electronic, 2326-7186 printed) is a journal of the International Research Center for the Mathematics and Mechanics of Complex Systems at the Università dell'Aquila, Italy.

Cover image: "Tangle” by $\odot$ John Horigan; produced using the Context Free program (contextfreeart.org).

PUBLISHED BY

7 mathematical sciences publishers

nonprofit scientific publishing

http://msp.org/

(C) 2016 Mathematical Sciences Publishers 
Special issue in honor of

Lucio Russo

Lucio Russo: A multifaceted life

Raffaele Esposito and Francesco dell'Isola

The work of Lucio Russo on percolation Geoffrey R. Grimmett

"Mathematics" and "physics" in the science of harmonics Stefano Isola

From quantum to classical world: emergence of trajectories in a quantum system Rodolfo Figari and Alessandro Teta

Propagation of chaos and effective equations in kinetic theory: a brief survey Mario Pulvirenti and Sergio Simonella

What decides the direction of a current? Christian Maes

A remark on eigenvalue perturbation theory at vanishing isolation distance Fiorella Barone and Sandro Graffi

Some results on the asymptotic behavior of finite connection probabilities in percolation Massimo Campanino and Michele Gianfelice

Correlation inequalities for the Potts model Geoffrey R. Grimmett

Quantum mechanics: some basic techniques for some basic models, I: The models Vincenzo Grecchi

Quantum mechanics: some basic techniques for some basic models, II: The techniques Vincenzo Grecchi

On stochastic distributions and currents

Vincenzo Capasso and Franco Flandoli

A note on Gibbs and Markov random fields with constraints and their moments Alberto Gandolfi and Pietro Lenarda

Quantum mechanics: light and shadows (ontological problems and epistemic solutions) Gianfausto Dell'Antonio

MEMOCS is a journal of the International Research Center for the Mathematics and Mechanics of Complex Systems at the Università dell' Aquila, Italy.

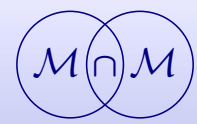

